\title{
Effects of circuit training interventions on bone metabolism markers and bone density of old women with osteopenia
}

\author{
Ki-Hong Kim', Hyang-Beum Lee ${ }^{2, *}$ \\ 'Department of Special Physical Education, Yong-In University, Yongin, Korea \\ ${ }^{2}$ Department of Physical Education, Yong-In University, Yongin, Korea
}

The purpose of this study is to propose an effective exercise intervention program to prevent and control osteopenia and osteoporosis that result from aging. To the end of this study, a circuit training intervention was performed for 8 weeks among participants with osteopenia who resided in Gyeonggi Province and were aged 65 and whose T-score of dual energy $X$-ray absorptiometry was between -1.0 and -2.5 . Based on the effect of this intervention on their bone metabolism markers and bone density, the following conclusions were derived: First, it turned out that the circuit training intervention had statistically significant interactive effects on bone metabolism markers (osteocalcin and deoxypyridinoline) of old women with osteopenia depending on the measuring tim- ing and groups. Second, it turned out that the circuit training intervention had statistically significant interactive effects on the bone density (T-score) depending on the measuring timing and groups. The conclusions above indicate that the circuit training intervention showed positive effects on bone metabolism markers and bone density of old women with osteopenia, being viewed as an effective intervention program applicable in order to prevent and control osteopenia and osteoporosis in line with bone density decrease.

Keywords: Circuit training, Osteopenia, Bone metabolism markers, Bone density, Old women

\section{INTRODUCTION}

As science and medical technology advanced, the general human lifespan has been extended. Accordingly, interests in methods to prevent chronic degenerative diseases that result from aging have increased among those who desire to enjoy a healthy old period of life.

Osteoporosis is one of the most common metabolic diseases that are connected to aging (Faibish et al., 2006). Osteopenia is the previous step of osteoporosis. As it causes bone mass decrease and structural changes, weakening the bone intensity and increasing the risk of fracture, osteopenia decreases the quality of human life (Ormsbee et al., 2014). Such bone mass decrease is distinctive among women compared to men. Bone mass increases gradually throughout the growth process until the mid-30s when it starts to reduce as much as $1 \%$ each year. As a woman goes into the time of menopause, the reduction rate is doubled with the risk of osteoporosis increasing (Ribom et al., 2004).

As osteopenia and osteoporosis deteriorate physical functions, the person becomes less capable of walking and the risk of injuries from a fall increases. An injury from a fall may lead to fracture and hospitalization. As a result, deterioration of osteopenia and osteoporosis causes loss of physical functions even to the point of death. Thus, effective solutions are to be developed (Shahin et al., 2010). One way of preventing and controlling osteopenia and osteoporosis is an exercise therapy to improve the bone density. For example, a type of training that induces physical stress to osseous tissues such as weight or load bearing exercise is recommended. This type of training delays the reduction of bone density and even increases bone mass (Rittweger et al., 2000). Bone mass is an index
${ }^{*}$ Corresponding author: Hyang-Beum Lee (iD https://orcid.org/0000-0001-5453-6335 Department of Physical Education, Yong-In University, 134 Yongindaehak-ro, Cheoin-gu, Yongin 17092, Korea

E-mail: hblee0908@naver.com

Received: December 28, 2018 / Accepted: February 6, 2019
This is an Open Access article distributed under the terms of the Creative Commons Attribution Non-Commercial License (http://creativecommons.org/licenses/by-nc/4.0/) which permits unrestricted non-commercial use, distribution, and reproduction in any medium, provided the original work is properly cited. 
that reflects the level of bone density. It is not only an important element for diagnosis of osteopenia and osteoporosis but also a predictive factor for the determine the risk of fracture. A bone density test is the best method to predict the risk of fracture at the moment, but the analysis of bone metabolism markers is also necessary in order to determine the extent of bone formation and loss if a main goal is to predict bone density in the future or to grasp metabolic transition of bones (Marshall et al., 1996). A bone metabolism marker is used to predict the bone loss rate and risk of fracture as well as to evaluate responses to osteoporosis treatment (Demers and Kleerekoper, 1994). Among bone metabolism markers, osteocalcin is utilized commonly as a bone formation index as it is composed in osteoblasts and combined with substrates of osteocytes (Akesson et al., 1995). One of the most commonly used bone resorption indexes is deoxypyridinoline in which collagen is discomposed and isolated by osteoclasts (Szulc et al., 2000).

One way to prevent osteopenia and osteoporosis by improving bone metabolism markers is repeated load bearing exercise. Since such load bearing exercise is to repeat monotonous motions, people often lose interests and give up that exercise in the middle (Liu and Latham, 2009). For exercise to continue for a long period of time on a regular basis, therefore, an intervention that can change bone metabolism markers and bone density is required. Among many types of exercise, circuit training presents a variety of exercise items compared to other training methods, and each item can be practiced within a short time. It is known that for this reason, the drop-out rate is lower than that in other types of training (Davis et al., 2000). If circuit training has the same training effects with repeated weight bearing or load bearing exercise which is known for the positive effects on bone metabolism markers and bone density, voluntary participation with interests in exercise can be induced. Improving bone metabolism markers and bone density in this manner will be able to contribute to the quality of life among old people.

Accordingly, this study aims to clarify the effects of circuit training interventions on bone metabolism markers and bone density of old women with osteopenia who seek to improve the quality of their old period of life and to propose an exercise intervention program effective for their independent life style.

\section{MATERIALS AND METHODS}

\section{Study subjects}

Subjects for this study were selected among women aged 65 or older who would visit a welfare center for the old located in Gyeo-
Table 1. Physical characteristics of subjects

\begin{tabular}{lcc}
\hline Characteristic & Exercise group $(\mathrm{n}=9)$ & Control group $(\mathrm{n}=10)$ \\
\hline Age $(\mathrm{yr})$ & $72.89 \pm 3.26$ & $71.30 \pm 3.77$ \\
Height $(\mathrm{cm})$ & $153.42 \pm 3.88$ & $154.73 \pm 3.90$ \\
Weight $(\mathrm{kg})$ & $58.40 \pm 3.62$ & $57.61 \pm 2.62$ \\
T-score (point) & $-1.76 \pm 0.41$ & $-1.66 \pm 0.38$
\end{tabular}

Values are presented as mean \pm standard deviation.

nggi Province. Subjects' T-score of dual energy X-ray absorptiometry (DEXA) was between -1.0 and -2.5, and they were diagnosed as osteopenia (World Health Organization, 2007). Those practicing regular physical activity or taking a certain medication, which might affect the research result, were excluded from this study. Prior to the circuit training intervention, the gist and purpose of this study were explained to subjects, and those who agreed to participate voluntarily filled out the agreement form. The intervention program was conducted among them, and during the experiment period, any physical activity or certain medication that might affect the experiment results were prohibited. Physical characteristics of study subjects are summarized in Table 1.

\section{Measured items and methods for measurement}

In order to examine the effects of the 8-week circuit training intervention on old women's bone metabolism markers and bone density, bone metabolism markers (osteocalcin and deoxypyridinoline) were measured and bone density was also analyzed based on the T-score.

Among bone metabolism markers, osteocalcin was analyzed through blood tests. For blood testing, a medical technologist collected bloods from subjects' forearm veins $b$ means of disposable injectors between 9 and 10 in the morning. ELSA-OSTEO (CIS Bio International, Gif-sur-Yvette Cedex, Paris, France) analysis kit was utilized with the radiation isotope as the indicator. The antigen antibody reactions were tracked by means of the immunoradiometric assay. For deoxypyridinoline, urine tests were conducted. After $8 \mathrm{hr}$ of empty stomachs, urine that was collected the second time was analysis. Pyrilinks-D (Metra Biosystems Inc., Mountain View, CA, USA) was utilized as the analysis kit with enzymes as the indicator. Antigen antibody reactions were analyzed by means of the enzyme linked immunosorbent assay. After analysis, measurements were corrected based on the urine creatinine values in order to remove errors related to kidney functions. The bone density was measured by means of dual energy X-ray absorptiometry (Lunar Radiation Corp., Madison, WI, USA). Subjects' bone density status was analyzed based on the T-score bone 
density standards presented by the World Health Organization (2007).

\section{Circuit training program}

The intervention program used in this study was conducted 3 times a week, 50 to 60 min each time, for 8 weeks in total according to the recommendation of American College of Sports Medicine (2014). For warm-up and warm-down, whole-body stretching was practiced. For the main exercise, the following items were practiced 12 to 15 times for 40 sec each time: Dumbbell Press, Dumbbell Lateral Raise, Dumbbell Curl, Dumbbell Overhead Triceps Extension, Push Up, Crunch, Back Extension, Lunge, and Squat. There was a resting time between items for $20 \mathrm{sec}$, and an intermission between sets was given for $2 \mathrm{~min}$. The exercise strength was between 11 and 14 according to the Borg scale.

\section{Data processing}

For data processing in this study, the average and standard deviation of results were calculated by means of IBM SPSS Statistics ver. 22.0 (IBM Co., Armonk, NY, USA). In addition, repeated measures of analysis of variance (ANOVA) were conducted in order to examine changes in the bone metabolism markers and bone density of old women with osteopenia after the circuit training intervention. The statistical significance of results was verified on the level of 0.05 . Since the significance of results was likely to be affected by the number of sample cases, Partial $\eta^{2}$ was calculated as the effect size indicating the relational strength.

\section{RESULTS}

\section{Changes in bone metabolism markers after the circuit training intervention}

As the variance of bone metabolism markers (osteocalcin, deoxypyridinoline) of old women with osteopenia depending on the circuit training intervention and the homogeneity of covariate matrices were verified, the osteogenesis index osteocalcin was as follows: Box $M=3.281, F(3,92,265.543)=0.953$, and $P=0.414$. The bone resorption index deoxypyridinoline was as follows: Box $M=3.303, F(3,92,265.543)=0.960$, and $P=0.410$. These results satisfied the criteria of homogeneity verification. In the verification of the Mauchly bulb formation assumption regarding the variance of the treatment period as well, Huynh-Feldt value of $\varepsilon$ was 1 for both osteocalcin and deoxypyridinoline, satisfying the criteria of the bulb formation assumption.

As the criteria of homogeneity and bulb formation assumptions were satisfied, repeated measures of ANOVA of bone metabolism markers were conducted after the circuit training intervention, and the results are presented in Table 2. As shown in Table 2, it turned out that the circuit training intervention had interactive effects on bone metabolism markers (osteocalcin and deoxypyridinoline) of old women with osteopenia depending on the measuring timing and groups.

\section{Changes in bone density (T-score) after the circuit training intervention}

As the variance of bone density (T-score) and the homogeneity of covariate matrices were examined among old women with osteopenia after the circuit training intervention, Box $M=0.902$, $F(3,92,265.543)=0.262, P=0.853$, which indicates that the criteria of the homogeneity assumption were satisfied. As the Mauchly bulb formation assumption was examined in relation to the variance of the treatment period as well, Huynh-Feldt $\varepsilon$ was 1 , which indicates that the criteria of the bulb formation assumption were satisfied.

As the criteria of homogeneity and bulb formation assumptions were satisfied, repeated measures of ANOVA of bone density (T-score) were conducted after the circuit training intervention, and the results are presented in Table 3. As shown in Table 3, it

Table 2. Changes in bone metabolism markers after the circuit training intervention

\begin{tabular}{lccccccc}
\hline Bone metabolism marker & Group & Pretest & Posttest & & F & $P$-value & $\eta p^{2}$ \\
\hline Osteocalcin & EG & $9.76 \pm 0.96$ & $12.83 \pm 0.89$ & Group & 10.101 & 0.005 & 0.373 \\
& CG & $9.78 \pm 1.10$ & $9.81 \pm 1.12$ & Time & $2,283.557$ & 0.001 & 0.993 \\
Deoxypyridinoline & & & & GxT & $2,193.729$ & 0.001 & 0.992 \\
& EG & $5.79 \pm 0.90$ & $5.60 \pm 0.82$ & Group & 0.112 & 0.742 & 0.007 \\
& CG & $5.55 \pm 0.93$ & $5.56 \pm 0.90$ & Time & 9.532 & 0.007 & 0.359 \\
& & & & GxT & 13.470 & 0.002 & 0.442 \\
\hline
\end{tabular}

Values are presented as mean \pm standard deviation.

EG, exercise group; CG, control group. 
Table 3. Changes in bone density (T-score) after the circuit training intervention

\begin{tabular}{cccclrrc}
\hline & Group & Pretest & Posttest & & $F$ & $P$-value & $\eta p^{2}$ \\
\hline T-score & EG & $-1.76 \pm 0.41$ & $-1.63 \pm 0.37$ & Group & 0.065 & 0.802 & 0.004 \\
(point) & CG & $-1.66 \pm 0.38$ & $-1.64 \pm 0.37$ & Time & 10.747 & 0.004 & 0.387 \\
& & & & $G \times T$ & 5.552 & 0.031 & 0.246
\end{tabular}

Values are presented as mean \pm standard deviation.

EG, exercise group; CG, control group.

turned out that the circuit training intervention had interactive effects on bone density (T-score) of old women with osteopenia depending on the measuring timing and groups.

\section{DISCUSSION}

In order to propose an exercise intervention program effective for prevention and control of osteopenia and osteoporosis, which result from aging, a circuit training intervention was performed for 8 weeks among participants with osteopenia who resided in Gyeonggi Province and were aged 65 or older and whose T-score of DEXA was between -1.0 and -2.5. The effects of this intervention on their bone metabolism markers and bone density were analyzed and discussed in comparison with previous studies.

\section{Changes in bone metabolism markers after the circuit training intervention}

Osseous tissues play an important role in terms of structure and function. In a human body, bones maintain the homeostasis of mineral matters, protect organs, and keep body postures. As such, bones are regulated, directly or indirectly, by physical strength such as compressive force, tension, shearing force, and torsion as well as hormones, physiologically active materials, and so forth (Mundy et al., 1999). The level of bone metabolism can be estimated by measuring bone metabolism markers through blood and urine tests of bone matrix components that are isolated upon bone resorption and bone formation as well as enzymes released from osteoclast and osteoblast (Greenspan et al., 1998). As a bone formation index, osteocalcin is commonly utilized as it is composed in osteoblasts and combined with substrates of osteocytes (Akesson et al., 1995). Among bone resorption indexes, deoxypyridinoline that is from which collagen is decomposed and isolated by osteoclast is most commonly used (Szulc et al., 2000). Load bearing exercise is recommended as a way of facilitating bone metabolism. In this regard, it is known that repeated load bearing exercise applying force to bones facilitates bone metabolism and enhances the density (Srinivasan et al., 2010). However, ordinary weight bearing exercise repeats monotonous motions and thus it does not last long enough despite the known effects and necessity of exercise. Compared to such ordinary repeated load bearing exercise, circuit training interventions can enhance the concentration of exercise as they present various exercise items and the duration of each item is relatively short. Accordingly, this study examines changes in bone metabolism markers among old women with osteopenia as circuit training is applied.

As changes in bone metabolism markers of old women with osteopenia were analyzed with a circuit training intervention applied, there was statistically significant difference in interactive effects on both osteocalcin and deoxypyridinoline depending on the measuring timing and groups. This result verifies the positive effects of a circuit training intervention. In other words, a circuit training intervention facilitated the bone metabolism of old women with osteopenia. The results above correspond to is are supported by the following previous research findings: Tartibian et al. (2011) found that long-term aerobic exercise increases osteocalcin of women after menopause; Iwamoto et al. (2004) found that treadmill exercise increases rats' osteocalcin and decreases deoxypyridinoline, improving the bone metabolism; Bergström et al. (2012) found that resistance and aerobic exercise training for 1 year decreased the density of bone resorption markers among women in the time of menopause. Such findings are thought to indicate that mechanical shock of a circuit training intervention on skeletons facilitated the bone metabolism.

Based on the findings above, it is thought that a circuit training intervention enhances the bone metabolism by inducing physical stimulation effectively with neither boring repetition of weight bearing exercise nor change of large-size devices or tools. Thus, circuit training can be an effective intervention to prevent and control osteoporosis among old women with osteopenia.

\section{Changes in bone density (T-score) after the circuit training intervention}

Osteoporosis among women causes the basal metabolic rate and bone density to decrease along with menopause. Osteoporosis not only causes pain but also decreases functional abilities, restricting independence in one's life (Lin and Lane, 2004). Besides, it is reported that one third of women after menopause suffer from osteoporosis, which indicates that osteoporosis is a critical health problem among women after menopause (Compston, 2001). Osteopenia is a phenomenon of aging in the previous step of osteoporosis. When the T-score is between -1.0 and -2.5 in DEXA, it 
is diagnosed as osteopenia (World Health Organization, 2007). One way to prevent and delay loss of bones is an exercise cure which is effective as it not only increases bone mass but also regulates risks of fracture and improves physical strength (Sherrington et al., 2008). In reflection of these facts, changes in bone density among old women with osteopenia were observed after the circuit training intervention was applied.

As changes in bone T-score of old women with osteopenia were analyzed with a circuit training intervention applied, there was statistically significant difference in interactive effects depending on the measuring timing and groups. This result verifies the statistical increase of the T-score. In other words, the circuit training intervention improved the T-score of bone density among old women with osteopenia. As a resistance exercise was applied to subjects aged between 55 and 74, men and women, the spinal bone density increased significantly according to Bemben and Bemben (2011). Chilibeck et al. (2005) also reported that a resistance exercise intervention applied to old men caused the bone density over the body to increase significantly. According to Daly et al. (2005), while the bone density decreased in the subject group of no exercise, there was no change in the bone density among subjects to whom a resistant exercise was applied. These findings support the result of this study. These findings stated above are thought to indicate that bone density increased as bones reacted to physical stimulation by the circuit training intervention.

The research results above indicate that a circuit training intervention applied to old women with osteopenia induces changes to the bone density similar to those of ordinary repeated weight bearing exercises, and thus that circuit training can be utilized as exercise intervention programs to delay and regulate bone loss among old women with osteopenia.

\section{CONFLICT OF INTEREST}

No potential conflict of interest relevant to this article was reported.

\section{REFERENCES}

Akesson K, Vergnaud P, Delmas PD, Obrant KJ. Serum osteocalcin increases during fracture healing in elderly women with hip fracture. Bone 1995;16:427-430.

American College of Sports Medicine. ACSM's guidelines for exercise testing and prescription. 9th ed. Philadelphia (PA): Lippincott Williams \& Wilkins; 2014.
Bemben DA, Bemben MG. Dose-response effect of 40 weeks of resistance training on bone mineral density in older adults. Osteoporos Int 2011; 22:179-186.

Bergström I, Parini P, Gustafsson SA, Andersson G, Brinck J. Physical training increases osteoprotegerin in postmenopausal women. J Bone Miner Metab 2012;30:202-207.

Chilibeck PD, Chrusch MJ, Chad KE, Shawn Davison K, Burke DG. Creatine monohydrate and resistance training increase bone mineral content and density in older men. J Nutr Health Aging 2005;9:352-353.

Compston JE. Sex steroids and bone. Physiol Rev 2001;81:419-447.

Daly RM, Dunstan DW, Owen N, Jolley D, Shaw JE, Zimmet PZ. Does high-intensity resistance training maintain bone mass during moderate weight loss in older overweight adults with type 2 diabetes? Osteoporos Int 2005;16:1703-1712.

Davis JM, Alderson NL, Welsh RS. Serotonin and central nervous system fatigue: nutritional considerations. Am J Clin Nutr 2000;72(2 Suppl): 573S-578S.

Demers LM, Kleerekoper M. Recent advances in biochemical markers of bone turnover. Clin Chem 1994;40(11 Pt 1):1994-1995.

Faibish D, Ott SM, Boskey AL. Mineral changes in osteoporosis: a review. Clin Orthop Relat Res 2006;443:28-38.

Greenspan SL, Parker RA, Ferguson L, Rosen HN, Maitland-Ramsey L, Karpf DB. Early changes in biochemical markers of bone turnover predict the long-term response to alendronate therapy in representative elderly women: a randomized clinical trial. J Bone Miner Res 1998; 13:1431-1438.

Iwamoto J, Shimamura C, Takeda T, Abe H, Ichimura S, Sato Y, Toyama Y. Effects of treadmill exercise on bone mass, bone metabolism, and calciotropic hormones in young growing rats. J Bone Miner Metab 2004;22:26-31.

Lin JT, Lane JM. Osteoporosis: a review. Clin Orthop Relat Res 2004;(425): 126-134.

Liu CJ, Latham NK. Progressive resistance strength training for improving physical function in older adults. Cochrane Database Syst Rev 2009; (3):CD002759.

Marshall D, Johnell O, Wedel H. Meta-analysis of how well measures of bone mineral density predict occurrence of osteoporotic fractures. BMJ 1996;312:1254-1259.

Mundy G, Garrett R, Harris S, Chan J, Chen D, Rossini G, Boyce B, Zhao $\mathrm{M}$, Gutierrez G. Stimulation of bone formation in vitro and in rodents by statins. Science 1999;286:1946-1949.

Ormsbee MJ, Prado CM, Ilich JZ, Purcell S, Siervo M, Folsom A, Panton L. Osteosarcopenic obesity: the role of bone, muscle, and fat on health. J Cachexia Sarcopenia Muscle 2014;5:183-192.

Ribom E, Ljunggren O, Piehl-Aulin K, Ljunghall S, Bratteby LE, Samuel- 
son G, Mallmin H. Muscle strength correlates with total body bone mineral density in young women but not in men. Scand J Med Sci Sports 2004;14:24-29.

Rittweger J, Beller G, Felsenberg D. Acute physiological effects of exhaustive whole-body vibration exercise in man. Clin Physiol 2000;20:134142.

Shahin D, Eltoraby E, Mesbah A, Houssen M. Insulin resistance in early untreated rheumatoid arthritis patients. Clin Biochem 2010;43:661-665.

Sherrington C, Whitney JC, Lord SR, Herbert RD, Cumming RG, Close JC. Effective exercise for the prevention of falls: a systematic review and meta-analysis. J Am Geriatr Soc 2008;56:2234-2243.

Srinivasan S, Ausk BJ, Prasad J, Threet D, Bain SD, Richardson TS, Gross
TS. Rescuing loading induced bone formation at senescence. PLoS Comput Biol 2010;6(9):e1000924.

Szulc P, Seeman E, Delmas PD. Biochemical measurements of bone turnover in children and adolescents. Osteoporos Int 2000;11:281-294.

Tartibian B, Hajizadeh Maleki B, Kanaley J, Sadeghi K. Long-term aerobic exercise and omega-3 supplementation modulate osteoporosis through inflammatory mechanisms in post-menopausal women: a randomized, repeated measures study. Nutr Metab (Lond) 2011;8:71.

World Health Organization. WHO scientific group on the assessment of osteoporosis at primary health care level. Summary meeting report. Brussels, Belgium, 5-7 May 2004. Geneva (Switzerland): World Health Organization; 2007. 